\title{
Focus prosody in Japanese-English early bilinguals: A pilot study
}

\author{
Albert Lee ${ }^{1}, Y i X u^{2}$ \\ ${ }^{1}$ Dept. of Linguistics and Modern Language Studies, The Education U. of Hong Kong, Hong Kong \\ ${ }^{2}$ Dept. of Speech, Hearing and Phonetic Sciences, University College London, United Kingdom \\ albertlee@eduhk.hk, yi.xu@ucl.ac.uk
}

\begin{abstract}
Typologically, some languages mark narrow focus with 'postfocus compression' (PFC) while others do not. For those which do, PFC is easily lost through bilingualism, at both societal and individual levels. At the societal level, when in contact with aPFC language (e.g. Southern Min), a likely +PFC language can lose this prosodic feature (e.g. Taiwan Mandarin) [1]; at the individual level, for bilingual speakers of a +PFC (e.g. Mandarin) and a-PFC (e.g. Southern Min) language, age plays a role in whether they can produce PFC in Mandarin or not [2].

In the latter case, however, the effect of contact and the apparent role of age cannot be teased apart. To better understand how individual characteristics (e.g. age) affect PFC realisation, this study analysed Japanese-English bilinguals, whose two languages are both + PFC. We recruited six early bilingual speakers to complete a speech production task [3] to see if they would produce PFC after narrow focus in Japanese. The results showed that the biracial speakers living in the United Kingdom manifested clear evidence of PFC, whereas another ethnic Japanese speaker who grew up in Japan but identified herself as English-dominant failed to produce PFC. The implications of these findings are discussed.
\end{abstract}

Index Terms: Bilingualism, English, Focus, Japanese

\section{Introduction}

\subsection{Focus markers in Japanese}

In Japanese, focus can be marked by syntactic (see [4] for a review), morphological (i.e. using the focus particles dake 'only' or mo 'too'), as well as prosodic means (reviewed in [5]). Previous studies reported that focus is marked by on-focus fundamental frequency $\left(f_{\mathrm{o}}\right)$ raising and post-focus compression of $f_{\mathrm{o}}$ range (PFC), and that PFC may be absent after a lexically unaccented word (e.g. [3], [6], [7]). There are also non- $f_{\mathrm{o}}$ cues such as duration and formant frequency [8].

Figure 1 shows smoothing spline ANOVA plots of $f_{\mathrm{o}}$ contours of various focus conditions from [3]. The upper panel represents a lexically all-accented sentence, where PFC is expected. Indeed, the initial focus contour (pink) is significantly lower than the corresponding neutral focus contour (lilac) in the post-focus region (second and third intervals from the left). The lower panel refers to the all-unaccented condition, where PFC is absent. Here the post-initial focus contour is higher than neutral focus, as though there is 'post-focus raising'. The role that lexical pitch accent plays in Japanese focus prosody was taken by [3] to suggest that PFC is not an all-or-nothing typological feature. Even for languages that are $+\mathrm{PFC}$, it does not follow that PFC is applied across the board; rather, its realisation can be conditional upon various factors, in this case, lexical accent.
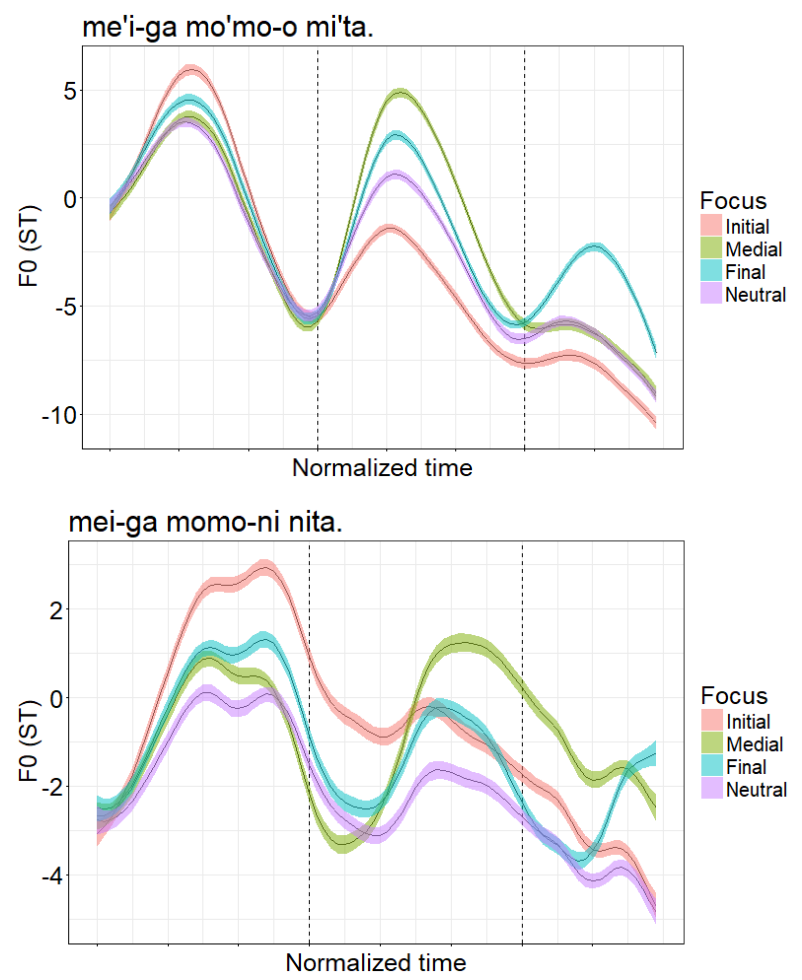

Figure 1: Prosodic realisation of narrow focus in Japanese by monolingual speakers (upper panel: allaccented sentence; lower panel: all-unaccented sentence, adapted from [3]). Dashed lines indicate word boundaries.

\subsection{Post-focus compression}

Since [1], PFC has been increasingly studied as a typological feature. It is a highly stylistic prosodic means of marking focus by compressing the $f_{\mathrm{o}}$ range and intensity of words after the focused words [1], [9]-[11]. It has been found to be present in many languages, but absent in others, and this dichotomy between +PFC and -PFC languages may have a historical source [12].

For those languages that do have PFC, it has been shown to be easily lost through bilingualism. On the societal level, Taiwan Mandarin has been found to be $-\mathrm{PFC}$, even though Beijing Mandarin is +PFC [1]. This was argued to be because Taiwan Mandarin is in contact with Southern Min (-PFC). At the individual level, usage was found to be associated with PFC realisation in Mandarin-Southern Min bilinguals in Mainland China [2]. These studies have shown that so much as PFC is typological in nature, its realisation is dynamic and depends on 
many factors such as bilingualism and lexical prosody as reviewed above.

The findings of [2] are important as they show how individual factors (i.e. usage) can mediate societal ones (i.e. societal bilingualism) in a case where a $+\mathrm{PFC}$ language is in contact with a-PFC one. What remains unclear, however, is whether the former is actually suppressing PFC in this situation, or if they 'resuscitate' what is otherwise inactive. To answer this question, it is important to first verify the assumption of whether PFC is guaranteed when two +PFC languages are in contact. A positive result would lend further support to [1] that PFC is lost through contact with $a-P F C$ language; otherwise, it would suggest that PFC is lost through language contact in a bilingual speech community in general. In turn, the former case would suggest that individual factors (e.g. usage) can 'resuscitate' PFC where it should be inactive, whereas the latter could point to a bigger role of individual factors in suppressing PFC realisation.

To this end, focus prosody in Japanese-English bilinguals would be a good test case. Like Japanese, English is a +PFC language [13]. In English, that a narrow focus is realized by expanding the $f_{\mathrm{o}}$ range of the on-focus stressed syllables, suppressing the $f_{\mathrm{o}}$ range of post-focus syllables, and leaving the $f_{\mathrm{o}}$ range of pre-focus syllables largely intact. Unlike in Japanese, PFC realisation in English seems to be insensitive to lexical prosody, as both stressed and unstressed syllables are subject to $f_{\mathrm{o}}$ range compression in the post-focus region. Thus the present study will shed light on the puzzle left behind by [2] as reviewed above.

\subsection{Research questions}

This paper seeks to investigate focus prosody in Japanese produced by Japanese-English bilinguals to shed light on the aforementioned issues. In doing so, we will test three hypotheses:

- H1: Japanese-English early bilinguals produce PFC;

- H2: Those with more exposure to Japanese are more likely to produce $\mathrm{PFC}$;

- H3: No overgeneralisation of PFC to all-unaccented sentences.

$\mathrm{H} 1$ is based on the assumption that PFC is lost only through contact with a $-P F C$ language, so that if both English and Japanese are +PFC [14], Japanese-English bilinguals would show PFC. H2 is related to [2] where younger speakers with more Mandarin usage produced PFC whereas the older speakers with less Mandarin usage did not. It would follow that bilingual speakers with more exposure to Japanese (e.g. by residing in Japan for longer) are more likely to produce PFC. For H3, as PFC is presumably easy to lose and in turn hard to acquire, it should be absent in cases where native monolinguals do not produce PFC [3], i.e. no overgeneralisation. The following production experiment was designed to test these hypotheses.

\section{Methods}

\subsection{Participants}

We conducted a Japanese production experiment in London with six Japanese-English early bilinguals. Table 1 summarises their demographic backgrounds:
Table 1: Demographic background of participants. 'Res JP' refers to number of years residing in Japan.

\begin{tabular}{|c|c|c|c|c|c|c|c|}
\hline & Age & Sex & Born & Grew up & Father & Mother & Res JP \\
\hline $\mathrm{AC}$ & 19 & $\mathrm{M}$ & 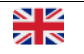 & NE & NE & - & 0 \\
\hline RA & 19 & $\mathrm{~F}$ & - & - 舆正 & $\bullet$ & $\bullet$ & 3 \\
\hline MM & 24 & $\mathrm{~F}$ & - & $\bullet$ & - & - & 18 \\
\hline $\mathrm{MN}$ & 27 & $\mathrm{~F}$ & • & 致。 & - & Ne & 11 \\
\hline SY & 43 & $\mathrm{~F}$ & 凮 & - & • & - & 11 \\
\hline KT & 23 & M & - & $\bullet$ & $\bullet$ & - & 9 \\
\hline
\end{tabular}

Biracial speakers $\mathrm{AC}$ and $\mathrm{MN}$ both grew up in the United Kingdom, but considered themselves equally proficient in Japanese and English. RA and KT also considered themselves equally proficient in both languages, though they had received most of their education in the UK. MN and SY attended international schools in Japan, but SY considered English her dominant language. All participants were remunerated a small sum for their time, and granted their written consent to being tested. This study has been approved by the UCL Research Ethics Committee [Ref. \# SHaPSetXU002].

\subsection{Stimuli}

A subset of the stimuli in [3] were adopted. Altogether 64 target sentences $(8$ accent conditions $\times 2$ sentence types $\times 4$ focus conditions) were elicited (see Table 1). There were four possible focus conditions for each target sentence, namely initial, medial, final, and neutral; since all target sentences contained three words, medial focus here is equivalent to penultimate focus in comparable studies. Each target sentence was repeated four times (i.e. 5 utterances). Focus was elicited with a leading question that contained a piece of inaccurate information in the word location of interest, and the speaker was to say the leading question and the target statement in pair in order to elicit narrow corrective focus. Neutral focus was elicited with a leading question that contained all accurate information (thus yielding no corrective focus).

Table 1: Target sentences used in this study (accented mora is marked with a following apostrophe).

\begin{tabular}{llll}
\hline & \multicolumn{1}{c}{ Word I } & Word II & \multicolumn{1}{c}{ Word III } \\
\hline \multirow{2}{*}{ A } & $\begin{array}{l}\text { me'i-ga } \\
\text { May-NOM }\end{array}$ & $\begin{array}{l}\text { mo'mo } \\
\text { thigh }\end{array}$ & $\begin{array}{l}\text {-o mi'ta } \\
\text {-ACC saw }\end{array}$ \\
\hline \multirow{2}{*}{$\mathrm{U}$} & $\begin{array}{l}\text { Mei-ga } \\
\text { Niece-NOM }\end{array}$ & $\begin{array}{l}\text { momo } \\
\text { peach }\end{array}$ & $\begin{array}{l}\text {-ni nita } \\
\text {-DAT resembled }\end{array}$ \\
\hline
\end{tabular}

From the 1,920 utterances collected, $42 \%$ were discarded due to minor hesitations and short pauses. Unlike the monolingual speakers in [3], the present bilingual speakers hesitated more with the less natural target sentences designed for laboratory experiments. This was despite the fact that they were all fluent and self-identified as having native competence in Japanese. To avoid unintended influences on pitch target realisation, we decided to discard all utterances that were not fluently produced for rigour's sake. For the purpose of answering our research questions, below we will present only results regarding the all-accented statement me'i-ga mo'mo-o $m i$ 'ta where PFC would always be expected for monolingual speakers, and those regarding the all-unaccented statement meiga- momo-ni nita where PFC is not expected. 
Recording took place in a sound-proofed room in London. All recording and data analysis procedures were identical to those in [3]. However, in this study, (i) participants were required to produce only half as many utterances to simplify the speaking task, and (ii) during pre-testing briefing they were asked to produce all individual words in the target sentences to ensure that they knew the correct lexical accent conditions of those words.

\section{Results}

Preliminary analysis of the results revealed considerable crossspeaker variability in $f_{\mathrm{o}}$ realisation of different focus conditions. Figure 2 shows that looking at all participants as a whole, the only $f_{\mathrm{o}}$ cue to focus that was consistently used was on-focus $f_{\mathrm{o}}$ peak raising. In the following sub-sections, individual speakers' production patterns will be analysed.

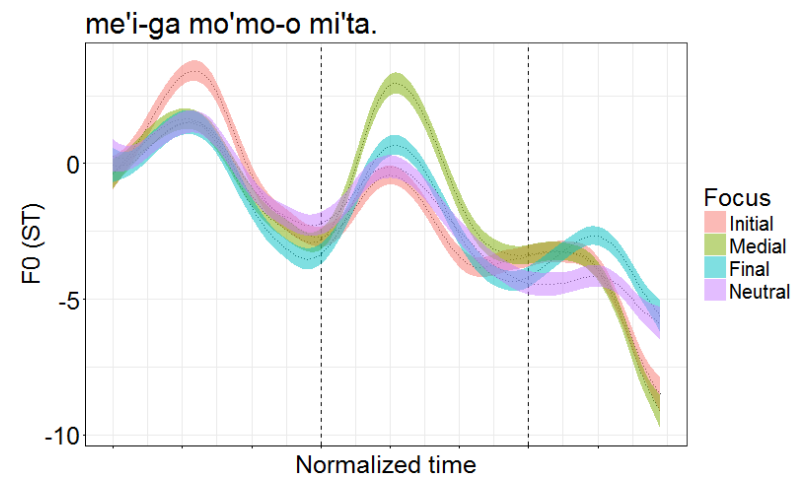

Figure 2: Prosodic realisation of narrow focus by early bilinguals (all participants).

\subsection{Successful production of PFC}

Three of the speakers (AC, KT, RA) successfully produced PFC in the appropriate contexts (i.e. where preceding words are accented). Figure 3 shows that in an all-accented sentence, both $\mathrm{AC}$ and RA clearly produced PFC, with the post-initial focus $f_{\mathrm{o}}$ contour (pink) significantly lower than the corresponding neutral focus contour (lilac) in the middle interval in the plot Even though RA showed a smaller degree of PFC, the difference between initial and neutral focus in post-focus $f_{\mathrm{o}}$ was significant.

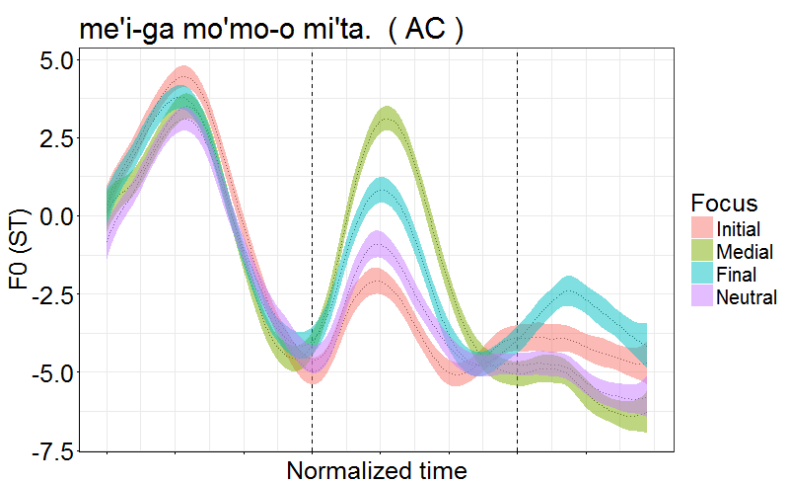

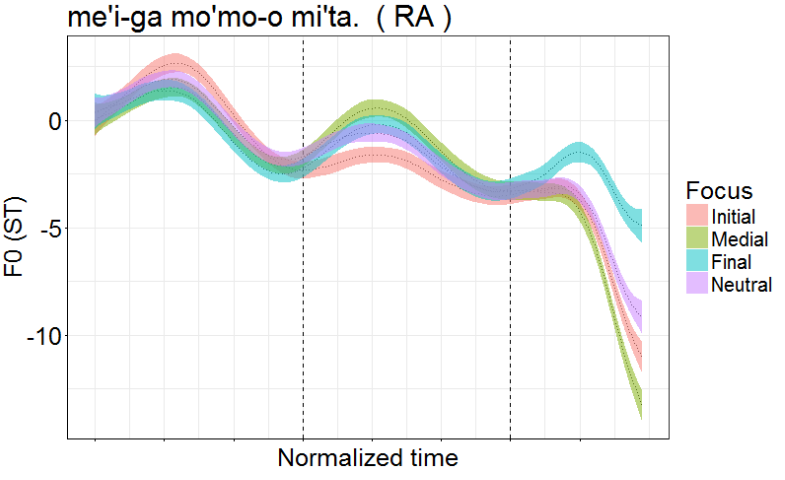

Figure 3: Examples of successful production of PFC (upper: $A C$; lower: $R A$ )

\subsection{Unsuccessful production of PFC}

The other speakers (MM, MN, SY) did not produce PFC where it is expected. Figure 4 shows that for MM post-focus $f_{\mathrm{o}}$ overlapped with the corresponding neutral focus contour in the middle interval in the plot, i.e. no PFC; for SY, post-focus $f_{\mathrm{o}}$ was even higher than neutral focus. For these speakers, the only

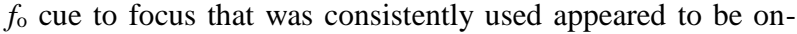
focus raising.
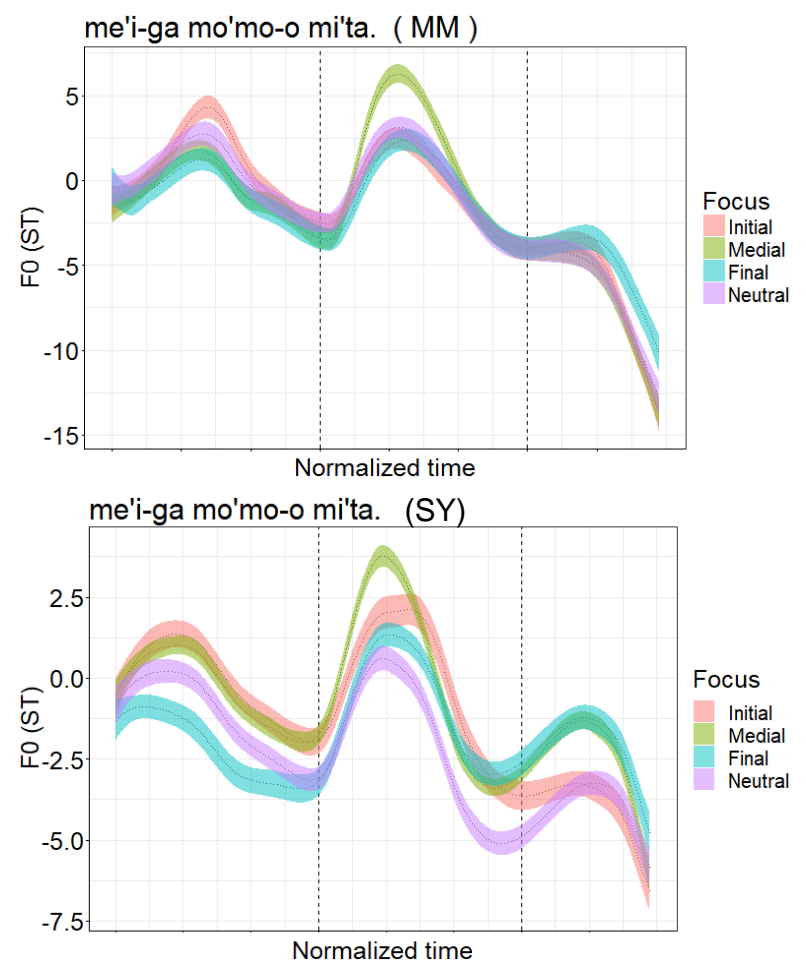

Figure 4: Examples of unsuccessful production of PFC (upper: MM; lower: SY).

\subsection{Overgeneralisation}

Finally, we also observed cases of overgeneralisation of PFC. Despite the fact that PFC should not be applied where preceding words do not bear the lexical pitch accent, one speaker always produced PFC in these sentences. AC, who successfully produced PFC in the right conditions (see §3.1), 
overgeneralised it to cases where PFC was not observed in native monolingual Japanese speakers [3]. Figure 5 shows that for initial focus (pink), post-focus $f_{\mathrm{o}}$ was significantly lower than the corresponding neutral focus contour, i.e. PFC.

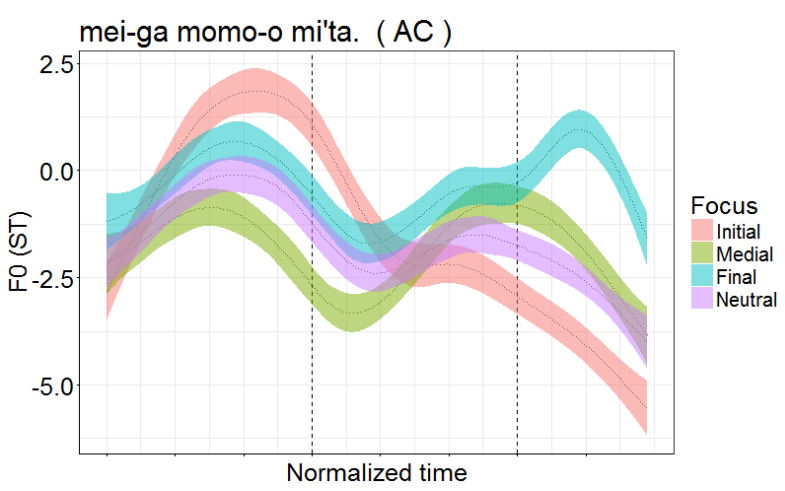

Figure 5: Example of overgeneralisation of PFC.

\section{Discussion}

In summary, the results of this study showed that there were some speakers who successfully acquired PFC as a focus marker, while others did not. This discrepancy does not support H1 ('Japanese-English early bilinguals produce PFC'). Our findings also appear to refute $\mathrm{H} 2$ ('Those with more exposure to Japanese are more likely to produce PFC'), as our $+\mathrm{PFC}$ speakers included biracial AC, and RA who had spent the least time living in Japan. Finally, in $\$ 3.3$ we showed that AC overgeneralised PFC to all-unaccented utterances where it is not required, thus rejecting $\mathrm{H} 3$ ('No overgeneralisation of PFC to all-unaccented sentences').

Although speaking two +PFC languages, namely English and Japanese, half of our early bilinguals did not manage to produce PFC in their Japanese speech. This thus rejected H1 and may possibly point to the view that PFC is not lost through contact with a -PFC language, but through contact with any language. If this is the case, a better way to put it would be that PFC is hard to acquire - growing up speaking two languages is challenging enough, so some difficult features that are not essential to communication (there are still on-focus raising, non- $f_{\mathrm{o}}$ cues, and non-prosodic cues to focus) may be omitted by some speakers.

In [2], PFC realisation was found to be associated with usage, which in turn would be conceivably related to factors such as language dominance in their context. While we did not manage to identify our participants' dominant language objectively, presumably those who had spent more time living in Japan would be more likely Japanese-dominant than biracial ones who grew up in an Anglophone country; but this was not the case. To our surprise, speaker SY who had received all her schooling in Japan and spoke only Japanese to her family, showed no trace of PFC in her speech. The only possible explanation may lie in her self-identification as Englishdominant, as she reportedly always spoke English except to her family. In contrast, biracial AC who had lived all his life in the UK would be expected to be English-dominant, but his Japanese speech showed the most consistent PFC. What kind of role self-identified language dominance (in turn attitude towards the target language) plays may be worth further investigation.
It was also interesting to note that $\mathrm{AC}$ overgeneralised $\mathrm{PFC}$ to cases where it was not expected, namely all-unaccented utterances. Although he seems to be rather successful in producing PFC in the right contexts, it turns out that he is not so successful in being fully native-like in this sense.

Needless to say, a larger sample size is necessary to verify the present findings. This is especially true for a study on bilingual speakers, who typically show great within- and crossspeaker variability. That said, this paper has presented first hand data on bilingual prosody, which is still a relatively understudied topic. Our findings may shed light on questions related to bilingualism, focus marking, as well as language acquisition.

\section{Acknowledgements}

This study was partially supported by the Japan Foundation Endowment Committee Grant (S272013) awarded to AL. An earlier version of this paper was presented at the $1^{\text {st }}$ International Conference on Prosodic Studies (ICPS1), Tianjin. 


\section{References}

[1] Y. Xu, S.-W.Chen, and B.Wang, "Prosodic focus with and without post-focus compression: A typological divide within the same language family?," Linguist. Rev., vol. 29, no. 1, pp. 131-147, 2012.

[2] Y. Chen, Y.Xu, and S.Guion-Anderson, "Prosodic realization of focus in bilingual production of Southern Min and Mandarin," Phonetica, vol. 71, no. 4, pp. 249-270, 2014

[3] A. Lee and Y.Xu, "Conditional realisation of post-focus compression in Japanese," in Proceedings of the 9th International Conference on Speech Prosody (SP2018), 2018, pp. 216-219.

[4] S. Ishihara, "Syntax-phonology interface," in Handbook of Japanese Phonetics and Phonology, H.Kubozono, Ed.Berlin Mouton de Gruyter, 2015.

[5] J. J. Venditti, K.Maekawa, and M. E.Beckman, "Prominence marking in the Japanese intonation system," in The Oxford Handbook of Japanese Linguistics, S.Miyagawa andM.Saito, Eds.New York, NY: Oxford University Press, 2008, pp. 456512.

[6] S. Ishihara, "Focus prosody in Tokyo Japanese wh-questions with lexically unaccented wh-phrases," in Proceedings of the 17th International Congress of Phonetic Sciences (ICPhS 2011), 2011, pp. 946-949.

[7] A. Lee and Y.Xu, "Revisiting focus prosody in Japanese," in Proceedings of the 6th International Conference on Speech Prosody (SP2012), 2012, pp. 274-277.

[8] K. Maekawa, "Effects of focus on duration and vowel formant frequency in Japanese," in Computing prosody: Computational models for processing spontaneous speech, Y.Sagisaka, W. N.Campbell, andN.Higuchi, Eds.New York, NY: Springer, 1997, pp. 129-153

[9] W. E. Cooper, S. J.Eady, and P. R.Mueller, "Acoustical aspects of contrastive stress in question-answer contexts," $J$ Acoust. Soc. Am., vol. 77, no. 6, pp. 2142-2156, 1985.

[10] S. J. Eady and W. E.Cooper, "Speech intonation and focus location in matched statements and questions.," J. Acoust. Soc. Am., vol. 80, no. 2, pp. 402-415, 1986.

[11] M. Sugahara, "Downtrends and post-focus intonation in Tokyo Japanese," University of Massachusetts, Amherst, Amherst, MA, 2003.

[12] Y. Xu, "Post-focus compression: Cross-linguistic distribution and historical origin," in Proceedings of the 17th International Congress of Phonetic Sciences (ICPhS 2011), 2011, pp. 152-155.

[13] Y. Xu and C. X.Xu, "Phonetic realization of focus in English declarative intonation," J. Phon., vol. 33, no. 2, pp. 159-197, 2005

[14] M. E. Beckman and J. B.Pierrehumbert, "Intonational structure in Japanese and English," Phonol. Yearb., vol. 3, pp. 255-309, 1986. 